\title{
Nonequilibrium Fluctuations in Micro-MHD Effects on Electrodeposition
}

\author{
Ryoichi Aogaki ${ }^{1}$ and Ryoichi Morimoto ${ }^{2}$ \\ ${ }^{1}$ Polytechnic University, Ryogoku, Sumida-ku, Tokyo, \\ 2Saitama Prefectural Okubo Water Filtration Plant \\ Shuku, Sakura-ku, Saitama-shi, Saitama, \\ Japan
}

\section{Introduction}

In copper electrodeposition under a magnetic field parallel to electrode, it is well known that though the drastic enhancement of deposition rate, a deposit surface receives specific levelling. This is because the Lorentz force generated by the interaction between magnetic field and electrolytic current induces a solution flow called magnetohydrodynamic (MHD) flow with micro-vortex called micro-MHD flow (Figs. 1 and 5a). The former is a laminar main flow, promoting mass transfer process (MHD effect), and the latter emerges inside the boundary layer, which often interacts with nonequilibrium fluctuations controlling electrochemical reactions.

MHD effect is exhibited by the following MHD current equation, where the promotion of mass transfer by the laminar flow is expressed by the increase of the average current density $\left\langle J_{z}\right\rangle$ (Aogaki et al., 1975).

$$
\left\langle J_{z}\right\rangle=H^{*} B_{0}{ }^{1 / 3} \theta_{\infty}^{*} 4 / 3
$$

where ' $<>$ ' denotes the average with regard to electrode surface, $H^{*}$ is a constant, $B_{0}$ is the magnetic flux density, and $\theta_{\infty}^{*}$ is the concentration difference between the bulk and surface. As one of the characteristic results of the electrodeposition in a parallel magnetic field, the interaction of the micro-MHD flow with nonequilibrium fluctuations called symmetrical fluctuations suppresses the three-dimensional (3D) nuclei with the order of $0.1 \mu \mathrm{m}$ in diameter to yield a flat surface (1st micro-MHD effect) (Aogaki, 2001; Morimoto et al., 2004). However, after long-term deposition in the same magnetic field, instead of leveling, semispherical secondary nodules with the order of $100 \mu \mathrm{m}$ in diameters are self-organized from two-dimensional (2D) nuclei together with the other nonequilibrium fluctuations, i.e., asymmetrical fluctuations (2nd micro-MHD effect) (Aogaki et al., 2008a, 2009a, 2010).

On the other hand, in a magnetic field vertical to electrode surface, minute vortexes vertical to the electrode surface emerge under a macroscopic tornado-like rotation called vertical MHD flow (Fig. 2); the formers come from 2D nucleation, whereas the latter is generated by the distortion of current lines in front of a disk electrode. As a result, a characteristic deposit with regular holes with about $100 \mu \mathrm{m}$ diameter called micro-mystery circles appears. 
Recently, using an electrode fabricated by the electrodeposition in a vertical magnetic field, the appearance of chirality in enantiomorphic electrochemical reactions was found, and it was suggested that the selectivity of the reactions comes from the chirality of the vortexes formed on the electrode (Mogi \& Watanabe, 2005; Mogi, 2008).

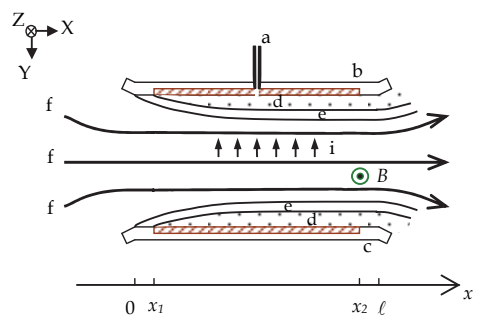

Fig. 1. MHD main flow and boundary layer. a, Luggin capillary; b, working electrode; $c$, counter electrode; $d$, diffusion layer; e, hydrodynamic boundary layer; $f$, streamline (Aogaki et al., 1975).

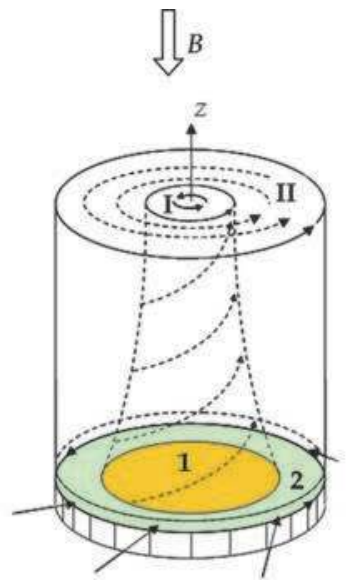

Fig. 2. Vertical MHD flow. 1, electrode; 2, electrode sheath; I, upward spiral flow; II, rotating flow; B, magnetic field (Sugiyama et al., 2004).

All these phenomena are attributed to the evolution or suppression process of nucleus by nonequilibrium fluctuations in a magnetic field. Generally, nucleation is classified into two types; one is 2D nucleation, i.e, expanding lateral growth, and the other is 3D nucleation, i.e., protruding vertical growth. These two types of nucleation result from different nonequilibrium fluctuations. Figure 3 shows two kinds of nonequilibrium fluctuations; one is asymmetrical fluctuation, which arises from electrochemical reactions in an electrical double layer. As shown in this figure, this fluctuation one-sidedly changes from an electrostatic equilibrium state toward cathodic reaction side, controlling 2D nucleation. The other is symmetrical fluctuation changing around an average value of its physical quantity in a diffusion layer. This fluctuation controls 3D nucleation. These concentration fluctuations are defined by 


$$
\begin{gathered}
c_{m}(x, y, z, t)^{a} \equiv C_{m}(x, y, z, t)-C_{m}^{*}(z, t) \\
c_{m}(x, y, z, t)^{s} \equiv C_{m}(x, y, z, t)-\langle C(x, y, z, t)\rangle
\end{gathered}
$$

where superscripts ' $a$ ' and ' $s$ ' imply the asymmetrical and symmetrical fluctuations, respectively. $C_{m}(x, y, z, t)$ and $C_{m}^{*}(z, t)$ are the concentration and the concentration in electrostatic equilibrium, respectively. $\langle C(x, y, z, t)\rangle$ is the average concentration over the electrode surface.

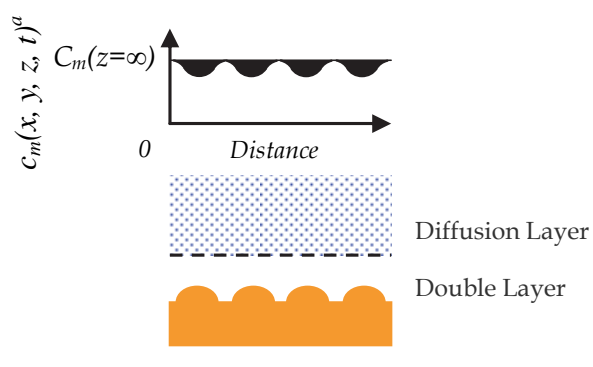

a

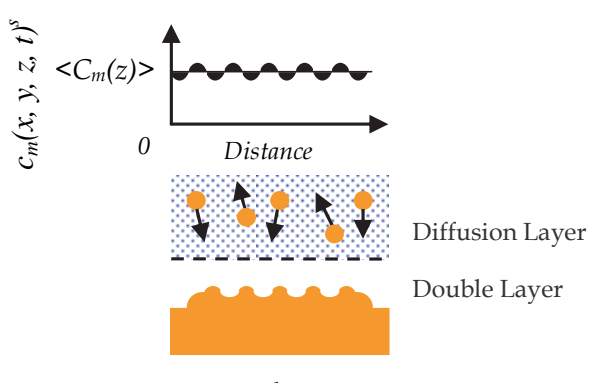

b

Fig. 3. Nonequilibrium fluctuations in electrodeposition. a, asymmetrical concentration fluctuation, which occurs in the electric double layer, controlling 2D nucleation in the scale of the order of $100 \mu \mathrm{m}$. b, symmetrical concentration fluctuation, which occurs in the diffusion layer, controlling 3D nucleation on 2D nuclei in the scale of the order of $0.1 \mu \mathrm{m} . C_{m}(z=\infty)$, bulk concentration; $\left\langle C_{m}(z)\right\rangle$, average concentration (Aogaki et al., 2010).

At the early stage of electrodeposition in the absence of magnetic field, there are two different kinds of the unstable processes of fluctuations. The first unstable process takes place in the electric double layer. In the case of electrodeposition without any specific adsorption, the overpotential of the double layer becomes negative with a positive gradient. Supposing that a minute 2D nucleus is accidentally formed in the diffuse layer of the double layer, at the top of the nucleus, due to the positive shifting of the potential, the double-layer overpotential decreases with the nucleation, so that with the unstable growth of the fluctuation, 2D nucleus is self-organized. As the reaction proceeds, outside the double layer, a diffusion layer emerges. In electrodeposition, due to the depletion of metallic ions at the electrode, the concentration gradient is also positive, so that the top of a 3D nucleus contacts with higher concentration than other parts. This means that the concentration overpotential decreases at the top of the nucleus. As a result, mass transfer is enhanced there, then the symmetrical fluctuations turn unstable, and the 3D nucleus is self-organized (Fig. 4a). In the presence of magnetic field, however, except for early stage, depending on the direction of magnetic field, nucleation proceeds in different ways; under a parallel magnetic field, as shown in Fig. $4 \mathrm{~b}$, from the interference of the micro-MHD flow to the concentration fluctuation in the diffusion layer, symmetrical fluctuations are always suppressed together with 3D nucleation (1st micro-MHD effect). 
In the secondary nodule formation after long-term deposition, it has been newly found that the flow mode of the solution changes from a laminar MHD flow to a convective microMHD flow induced by the asymmetrical fluctuations, so that the diffusion layer thickness slowly decreases with time, increasing electrolytic current. The mass transfer to 2D nuclei is thus enhanced, and secondary nodules are self-organized (2nd micro-MHD effect). Figure 5 schematically exhibits the change in the flow mode.

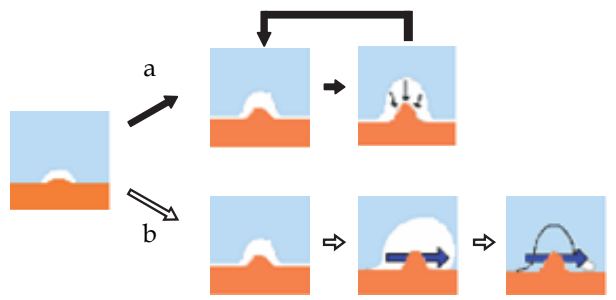

Fig. 4. Disturbance of symmetrical concentration fluctuation around a 3D nucleus by microMHD flow. a, without magnetic field, positive feedback process; $b$, with magnetic field, suppression of fluctuation by micro-MHD flow.

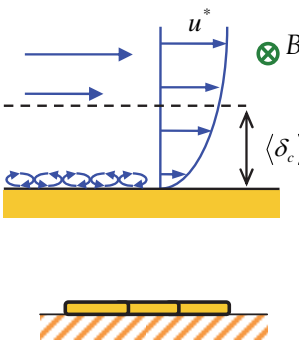

a

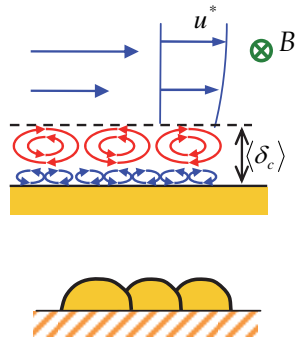

$\mathrm{b}$

Fig. 5. Change in the flow mode from laminar one (a) to convective one (b). $u^{*}$, velocity; $B$, magnetic flux density; $\left\langle\delta_{c}\right\rangle$, convective-diffusion layer thickness..

In a vertical magnetic field, for the appearance of chirality in vortex motion, ionic vacancy formed with electrodeposition plays an important role; as shown in Fig. 6, ionic vacancy is a vacuum void with a diameter of ca. $1 \mathrm{~nm}$ surrounded by ionic cloud (Aogaki, 2008b; Aogaki et al., 2009b), which expands the distance between solution particles, decreasing their interaction as a lubricant. In Fig. 7, it is shown that the vacancy generation during electrodeposition yields two kinds of electrode surfaces; a usual rigid surface with friction under a downward spiral flow of vortex, and a frictionless free surface covered with the vacancies under an upward spiral flow. This is because at the bottom of the downward flow, generated vacancies are swept away from the center, whereas under the upward flow, they are gathered to the center of the bottom. Theoretical examination suggests that the vortex rotation on the free surface is opposite to that on the rigid surface. As shown in Fig. 8, in a system rotating counterclockwise from a bird view, on the rigid surface, due to friction only a downward counterclockwise flow is permitted, while on a free surface, due to slipping of solution, only an upward clockwise flow is permitted. 


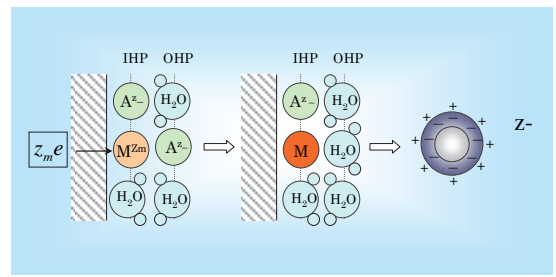

a

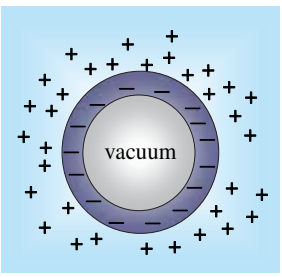

b

Fig. 6. Ionic vacancy. a, formation process, b, structure (Aogaki, 2008b). IHP, inner Helmholtz plane; OHP, outer Helmholtz plane; $\mathrm{M}^{\mathrm{zm}+}$, metallic ion; $\mathrm{A}^{\mathrm{z}-}$, counter anion.

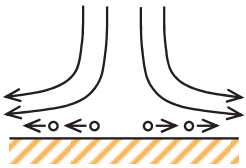

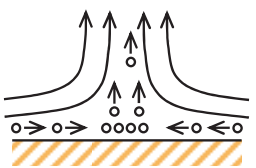

$\mathrm{b}$

Fig. 7. Formation of free and rigid surfaces by vacancies. $\odot$, Vacancy; a, rigid surface exposed without vacancies; b, free surface covered with vacancies (Aogaki et al., 2009c).

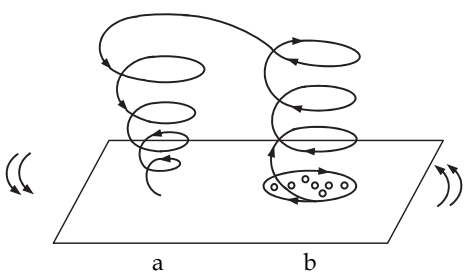

Fig. 8. Two kinds of vortexes on rigid and free surfaces in a counterclockwise rotating system from a bird view. ○, Vacancy; a, rigid surface; b, free surface (Aogaki et al., 2009c).

In such a system, not always magnetic field, but also macroscopic rotation such as vertical MHD flow and system rotation mentioned above are required; the magnetic field generates micro-MHD vortexes, and the macroscopic rotation, as shown in Fig. 9, bestows rotation direction and precession on them, which induces the interference of the vortexes with the concentration fluctuations. On the free surface of 2D nucleus, the metallic ions deposit in keeping the clockwise motion, yielding micro-mystery circles with chiral screw dislocations. This is the process of the formation of micro-mystery circle with chiral structure. On the rigid surface of 2D nucleus, due to friction of the electrode surface, a stationary diffusion layer is formed. Inside the static diffusion layer, in a fractal-like way, 3D nucleation induces smaller micro-MHD vortexes of symmetrical fluctuation, creating concentric deposits called nano-mystery circles. In the following sections, the roles of these nonequilibrium fluctuations will be more precisely elucidated. 


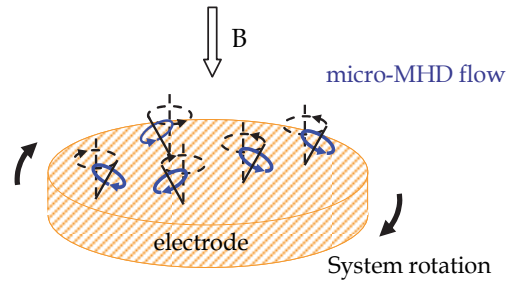

a

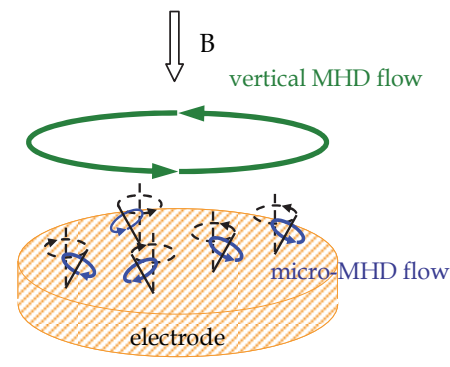

b

Fig. 9. Precession of micro-MHD flows. $a$, by system rotation; b, by vertical MHD flow.

\section{Instability in electrochemical nucleation}

\subsection{The first instability occurring in 2D nucleation}

Assuming that a minute 2D nucleus is accidentally formed in the diffuse layer belonging to electric double layer, we can deduce the first instability of asymmetrical fluctuations (Aogaki, 1995). The electrochemical potential fluctuation of metallic ion at the outer and inner Helmholtz planes (OHP, IHP) of the nucleus peak is, as will be shown in Eq. (15), expressed by the electrostatic potentials and the concentration overpotential in the electric double layer. The electrostatic potential fluctuation at the top of the nucleus $\phi_{2}\left(x, y, \zeta^{a}, t\right)^{a}$ in the diffuse layer is written by the potential fluctuation at the substrate $\phi_{2}(x, y, 0, t)^{a}$ and the potential fluctuation varied by the nucleus $L_{\phi}^{a} \zeta(x, y, t)^{a}$,

$$
\phi_{2}\left(x, y, \zeta^{a}, t\right)^{a}=\phi_{2}(x, y, 0, t)^{a}+L_{\phi}^{a} \zeta(x, y, t)^{a}
$$

where $\zeta^{a}$ is the surface height fluctuation of the 2D nucleus, and $L_{\phi}^{a}$ is the average potential gradient in the diffuse layer, defined by (Aogaki, 1995)

$$
L_{\phi}^{a}=-\frac{\left\langle\Phi_{2}\right\rangle}{\lambda}
$$

where $\lambda$ is the Debye length, and $\left\langle\Phi_{2}\right\rangle$ is the average potential fluctuation in the diffuse layer. In the case of deposition at early stage, as shown in Figs. 10 and 12, due to cathodic polarization, the average diffuse layer overpotential $\left\langle\Phi_{2}\right\rangle$ takes a negative value $\left\langle\Phi_{2}\right\rangle<0$ for no specific adsorption or aniodic specific adsorption, and takes a positive value $\left\langle\Phi_{2}\right\rangle>0$ for cationic specific adsorption, so that the average potential gradient in the diffuse layer $L_{\phi}^{a}$ becomes positive and negative, respectively. From Eq. (4a), the difference of the potential fluctuation at the OHP between the top and bottom of the nucleus is thus given by

$$
\Delta \phi_{2}\left(x, y, \zeta^{a}, t\right)^{a}=L_{\phi}^{a} \zeta(x, y, t)^{a}
$$


where

$$
\Delta \phi_{2}\left(x, y, \zeta^{a}, t\right)^{a} \equiv \phi_{2}\left(x, y, \zeta^{a}, t\right)^{a}-\phi_{2}(x, y, 0, t)^{a}
$$

In the same way as Eq. (5), the difference of the concentration fluctuation in the diffuse layer is expressed by

$$
\Delta c_{m}\left(x, y, \zeta^{a}, t\right)^{a}=L_{m}^{a} \zeta(x, y, t)^{a}
$$

where $L_{m}^{a}$ is the average concentration gradient in the diffuse layer, defined by (Aogaki, 1995)

$$
L_{m}^{a}=-\frac{z_{m} F}{\lambda R T} C_{m}^{*}(0, t)\left\langle\Phi_{2}\right\rangle
$$

where $R$ is the universal gas constant, $T$ is the absolute temperature, $z_{m}$ is the charge number, $F$ is Faraday constant, and

$$
\Delta c_{m}\left(x, y, \zeta^{a}, t\right)^{a} \equiv c_{m}\left(x, y, \zeta^{a}, t\right)^{a}-c_{m}(x, y, 0, t)^{a}
$$

Since both fluctuations are in the Boltzmann equilibrium in the diffuse layer, from Eqs. (4b) and (7b), the following relationship between $L_{m}^{a}$ and $L_{\phi}^{a}$ is obtained

$$
L_{m}^{a}=-\frac{z_{m} F}{R T} C_{m}^{*}(0, t) L_{\phi}^{a}
$$

On the other hand, the concentration overpotential is written by the Nernst equation.

$$
H\left(x, y, \zeta^{a}, t\right)=\frac{R T}{z_{m} F} \ln \left\{\frac{C_{m}\left(x, y, \zeta^{a}, t\right)}{C_{m}^{*}(z=\infty)}\right\}
$$

where $C_{m}^{*}(z=\infty)$ is the bulk concentration. From Eq. (2), the concentration at the top of the projection is written as

$$
C_{m}\left(x, y, \zeta^{a}, t\right)=C_{m}^{*}(0, t)+c_{m}\left(x, y, \zeta^{a}, t\right)^{a}
$$

Under the condition

$$
\left|c_{m}\left(x, y, \zeta^{a}, t\right)^{a}\right|<<C_{m}^{*}(0, t)
$$

Eq. (10) leads to the concentration overpotential fluctuation 


$$
\delta H\left(x, y, \zeta^{a}, t\right)^{a}=\frac{R T}{z_{m} F} \frac{c_{m}\left(x, y, \zeta^{a}, t\right)^{a}}{C_{m}^{*}(0, t)}
$$

where the approximation

$$
C_{m}^{*}\left(x, y, \zeta^{a}, t\right) \approx C_{m}^{*}(0, t)
$$

is used. Therefore, expanding the potential area to Helmholtz layer, we obtain the difference of the electrochemical potential fluctuation between the top and bottom of the nucleus.

$$
\Delta \delta \bar{\mu}_{m}\left(x, y, \zeta^{a}, t\right)^{a}=z_{m} F\left\{\Delta \phi_{1}(x, y, t)^{a}+\Delta \phi_{2}\left(x, y, \zeta^{a}, t\right)^{a}\right\}+\frac{R T}{C_{m}^{*}(0, t)} \Delta c_{m}\left(x, y, \zeta^{a}, t\right)^{a}
$$

$\phi_{1}(x, y, t)^{a}$ and $\phi_{2}(x, y, z, t)^{a}$ are the fluctuations of the electric potentials at the inner Helmholtz plane (IHP) (Helmholtz layer overpotential) and outer Helmholtz plane (OHP) (diffuse layer overpotential), respectively. Substitution of Eqs. (5) and (7a) into Eq. (15) with Eq. (9) leads to the cancellation of $\Delta \phi_{2}\left(x, y, \zeta^{a}, t\right)^{a}$ and $\Delta c_{m}\left(x, y, \zeta^{a}, t\right)^{a}$, so that only the term of the Helmholtz layer overpotential $\Delta \phi_{1}(x, y, t)^{a}$ survives. i.e.,

$$
\Delta \delta \bar{\mu}_{m}\left(x, y, \zeta^{a}, t\right)^{a}=z_{m} F \Delta \phi_{1}(x, y, t)^{a}
$$

$\phi_{1}(x, y, t)^{a}$ and $\phi_{2}\left(x, y, \zeta^{a}, t\right)^{a}$ are related by the differential double-layer potential coefficient $\left(\partial\left\langle\Phi_{1}\right\rangle / \partial\left\langle\Phi_{2}\right\rangle\right)_{\mu}$ (Aogaki, 1995).

$$
\phi_{1}(x, y, t)^{a}=\left(\frac{\partial\left\langle\Phi_{1}\right\rangle}{\partial\left\langle\Phi_{2}\right\rangle}\right)_{\mu} \phi_{2}\left(x, y, \zeta^{a}, t\right)^{a}
$$

where it should be noted that $\left\langle\Phi_{1}\right\rangle$ and $\left\langle\Phi_{2}\right\rangle$ denote the average values of the asymmetrical overpotential fluctuation of the Helmholtz and diffuse layers $\left\langle\phi_{1}(x, y, t)^{a}\right\rangle$ and $\left\langle\phi_{2}\left(x, y, \zeta^{a}, t\right)^{a}\right\rangle$, respectively. The subscript $\mu$ suggests that chemical potentials (activities) of the components are kept constant. Therefore, $\Delta \phi_{1}(x, y, t)^{a}$ is expressed by

$$
\Delta \phi_{1}(x, y, t)^{a}=\left(\frac{\partial\left\langle\Phi_{1}\right\rangle}{\partial\left\langle\Phi_{2}\right\rangle}\right)_{\mu} \Delta \phi_{2}\left(x, y, \zeta^{a}, t\right)^{a}
$$

Substituting Eq. (18) into Eq. (16), we have

$$
\Delta \delta \bar{\mu}_{m}\left(x, y, \zeta^{a}, t\right)^{a}=z_{m} F\left(\frac{\partial\left\langle\Phi_{1}\right\rangle}{\partial\left\langle\Phi_{2}\right\rangle}\right)_{\mu} \Delta \phi_{2}\left(x, y, \zeta^{a}, t\right)^{a}
$$



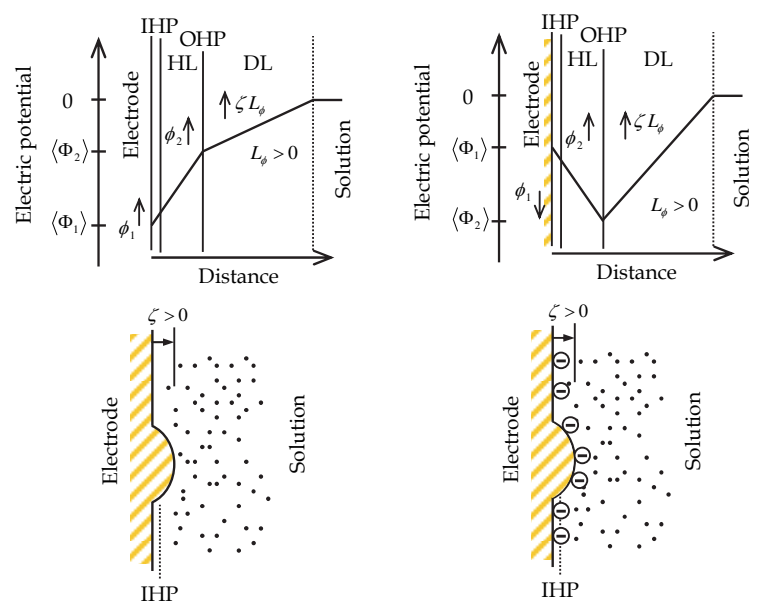

b

Fig. 10. Electrostatic potential distribution in the electric double layer. a; the case when specific adsorption is weak or absent, $b$; the case when anionic specific adsorption is strong. HL, Helmholtz layer; DL, diffuse layer.

The sign of the difference of the electrochemical potential fluctuation is determined by the difference of the potential fluctuation in the diffuse layer and the differential double-layer potential coefficient. As shown in Fig.10, in the case where no specific adsorption or anionic specific adsorption takes place, since the former is positive in the early stage of deposition (Eq. (5)), the sign of the electrochemical potential fluctuation depends on the latter value. When the specific adsorption of anion is absent or weak, i.e., $\left(\partial\left\langle\Phi_{1}\right\rangle / \partial\left\langle\Phi_{2}\right\rangle\right)_{\mu}>0$ is fulfilled, $\Delta \delta \bar{\mu}_{m}\left(x, y, \zeta^{a}, t\right)^{a}$ becomes positive. In view of the cathodic negative polarization in the diffuse layer, this means that at the top of the peak, the reaction resistance decreases, so that the nucleation turns unstable. In the case of strong specific adsorption of anion, due to the minimum point of the potential at the OHP shown in Fig.10b, on the contrary, $\left(\partial\left\langle\Phi_{1}\right\rangle / \partial\left\langle\Phi_{2}\right\rangle\right)_{\mu}<0$ is derived. As a result, the difference of the electrochemical-potential fluctuation in Eq. (19) becomes negative, which heightens the reaction resistance, leading to stable nucleation. When cationic specific adsorption occurs, as shown in Fig. 12b, due to negative potential gradient, $\Delta \phi_{2}\left(x, y, \zeta^{a}, t\right)^{a}$. becomes negative (Eq. (5)). Since cation does not yield intense specific adsorption, the potential distribution does not have a maximum point, so that $\left(\partial\left\langle\Phi_{1}\right\rangle / \partial\left\langle\Phi_{2}\right\rangle\right)_{\mu}>0$ is held. Therefore, $\Delta \delta \bar{\mu}_{m}\left(x, y, \zeta^{a}, t\right)^{a}<0$ leads to stable nucleation. Namely, at early stage, specific adsorption always suppresses 2D nucleation.

Without strong adsorption of anion or cation, the deposition process is accelerated, so that the asymmetrical fluctuation turns unstable, finally the $2 \mathrm{D}$ nucleus is self-organized. It is concluded that the asymmetrical fluctuations control the total electrode reaction, and the total electrolytic current increases. 


\subsection{The second instability in 3D nucleation}

As the reaction proceeds, outside the double layer; a diffusion layer is simultaneously formed, where the second instability occurs. According to the preceding paper (Aogaki et al., 1980), Fig. 11 shows the potential distribution in the diffusion layer, where an embryo of 3D nucleus is supposed to emerge. Since in the diffusion layer, due to metal deposition, the average concentration gradient of the metallic ion $L_{m}$ becomes positive, the difference of the concentration fluctuation between the top and bottom of the embryo becomes positive.

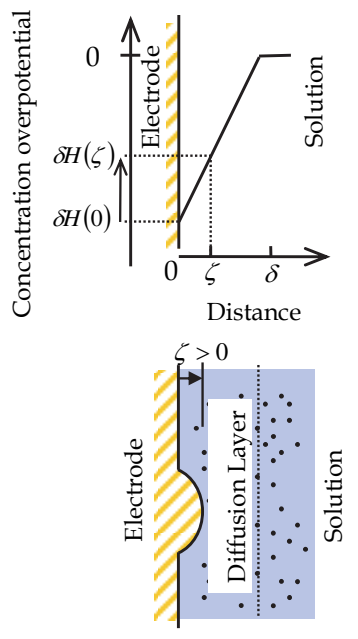

Fig. 11. Concentration distribution of metallic ion in the diffusion layer.

$$
\Delta c_{m}\left(x, y, \zeta^{s}, t\right)^{s}=L_{m} \zeta(x, y, t)^{s}(>0)
$$

where $\zeta^{s}$ is the surface height fluctuation of 3D nucleus. As will be discussed later, with the average thickness of the convective-diffusion layer $\left\langle\delta_{c}\right\rangle(>0)$ and the concentration difference between the bulk and surface $\theta_{\infty}^{*}(>0)$, the average concentration gradient of the diffusion layer is written by

$$
L_{m} \equiv \frac{\theta_{\infty}^{*}}{\left\langle\delta_{c}\right\rangle}(>0)
$$

According to Eqs. (3) and (13), for the symmetrical fluctuations, it is held that the difference of the concentration overpotential is also positive in the following,

$$
\Delta \delta H\left(x, y, \zeta^{s}, t\right)^{s}=\frac{R T}{z_{m} F\left\langle C_{m}(x, y, 0, t)\right\rangle} \Delta c_{m}\left(x, y, \zeta^{s}, t\right)^{s}(>0)
$$

where $\Delta \delta H^{s}$ is defined by the difference of the fluctuation between the top and the bottom of the nucleus 


$$
\Delta \delta H\left(x, y, \zeta^{s}, t\right)^{s} \equiv \delta H\left(x, y, \zeta^{s}, t\right)^{s}-\delta H(x, y, 0, t)^{s}
$$

Since the concentration overpotential takes a negative value for metal deposition, this means that at the top of the nucleus, the concentration overpotential decreases, accelerating instability, i.e., the following unstable condition is always fulfilled.

$$
\Delta \delta H\left(x, y, \zeta^{s}, t\right)^{s}>0
$$

Since the concentration gradient is positive, the top of the 3D nucleus contacts with higher concentration than other parts. Namely, the concentration overpotential decreases there, and mass transfer is enhanced. As a result, the symmetrical fluctuations always turn unstable, and the 3D nucleus is self-organized (Fig. 4a). However, in a magnetic field, since the microMHD flows interfere with the concentration fluctuation and disturb it, the 3D nucleation is resultantly suppressed together with not always the symmetrical concentration fluctuation but also the micro-MHD flow (1st micro-MHD effect)(Fig. 4b).

\subsection{The third instability in secondary nodule formation}

At the later stage of deposition, a grown 2D nucleus protrudes out of the double layer into the diffusion layer, which means that the nucleus develops under the same situation as that of 3D nucleation discussed above. At the same time, rate-determining step is changed from electron-transfer in the electric double layer to mass transfer in the diffusion layer, and expressed by the concentration overpotential; instability arises from the fluctuation of the concentration overpotential, $\delta H^{a}$ around the 2D nucleus, and the difference of the fluctuation between the top and the bottom of the nucleus $\Delta \delta H^{a}$ is defined by

$$
\Delta \delta H\left(x, y, \zeta^{a}, t\right)^{a} \equiv \delta H\left(x, y, \zeta^{a}, t\right)^{a}-\delta H(x, y, 0, t)^{a}
$$

Though $\Delta \delta H^{a}$ is expressed by Eq. (13), i.e.,

$$
\Delta \delta H\left(x, y, \zeta^{a}, t\right)^{a}=\frac{R T}{z_{m} F C_{m}^{*}(0, t)} \Delta c_{m}\left(x, y, \zeta^{a}, t\right)^{a}
$$

the difference of the concentration fluctuation is given not by $L_{m}^{a}$ but by $L_{m}$.

$$
\Delta c_{m}\left(x, y, \zeta^{a}\right)^{a}=L_{m} \zeta(x, y, t)^{a}(>0)
$$

Due to the positive values of $L_{m}$ and $\Delta c_{m}\left(x, y, \zeta^{a}\right)^{a}, \Delta \delta H\left(x, y, \zeta^{a}, t\right)^{a}$ in Eq. (26) becomes positive. Since cathodic polarization gives negative concentration overpotential, this indicates the decrease of the overpotential at the top of the 2D nucleus. Namely, from the same reason as the second instability, the unstable condition for $2 \mathrm{D}$ nucleation in the diffusion layer is always fulfilled. In view of the fact that the 2D nucleation arises from the electrode reaction process in the double layer, this unstable condition must be rewritten by the parameters of the double layer. With the ohmic drop disregarded, assuming that the total overpotential is kept constant, we can derive the following relationship between the fluctuations of the electrochemical potentials at the double layer and the diffusion layer. 


$$
\Delta \delta \bar{\mu}_{m}\left(x, y, \zeta^{a}, t\right)^{a}=-z_{m} F \Delta \delta H\left(x, y, \zeta^{a}, t\right)^{a}
$$

As a result, it is concluded that $\Delta \delta \bar{\mu}_{m}\left(x, y, \zeta^{a}, t\right)^{a}<0$ is the unstable condition for the secondary nodule formation from $2 \mathrm{D}$ nuclei in the diffusion layer. This condition also corresponds to the stable condition in the first instability of 2D nucleation. As shown in Fig. 12a, according to Eq. (19), for an anionic adsorbent, the positive difference $\Delta \phi_{2}^{a}(>0)$ in Eq. (5) from the negative overpotential $\left\langle\Phi_{2}\right\rangle$, and the negative value of the differential double layer potential coefficient $\left(\partial\left\langle\Phi_{1}\right\rangle / \partial\left\langle\Phi_{2}\right\rangle\right)_{\mu}(<0)$ due to strong specific adsorption give the unstable condition $\Delta \delta \bar{\mu}_{m}\left(x, y, \zeta^{a}, t\right)^{a}<0$. For a cationic adsorbent, since usually cation does not yield strong specific adsorption, as shown in Fig. 12b, negative difference $\Delta \phi_{2}^{a}(<0)$ in Eq. (5) from the positive overpotential $\left\langle\Phi_{2}\right\rangle$, and the positive value of $\left(\partial\left\langle\Phi_{1}\right\rangle / \partial\left\langle\Phi_{2}\right\rangle\right)_{\mu}(>0)$ due to weak specific adsorption lead to the same unstable condition $\Delta \delta \bar{\mu}_{m}\left(x, y, \zeta^{a}, t\right)^{a}<0$. Namely, after long-term deposition, whether adsorbent is anionic or cationic, specific adsorption induces unstable secondary nodule formation.
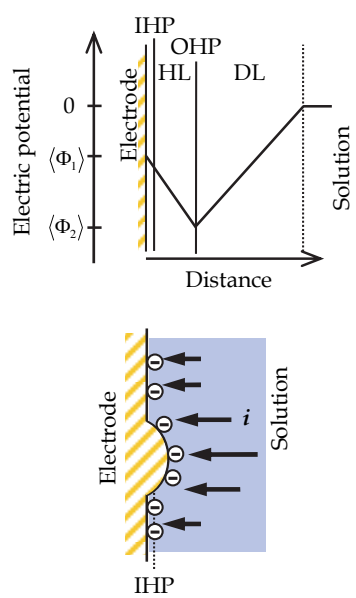

a
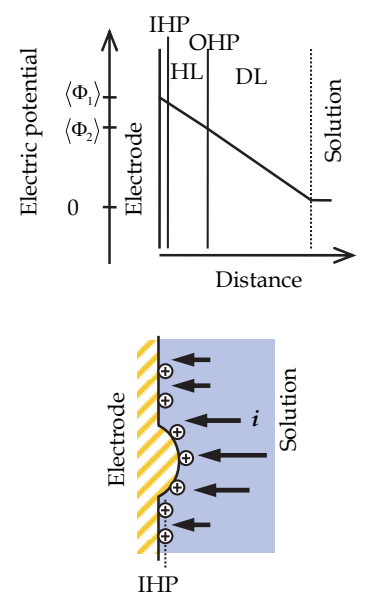

b

Fig. 12 Potential distribution in the electric double layer by specific adsorption. a, anionic adsorbent; $\mathrm{b}$, cationic adsorbent.

\section{First and second micro-MHD effects in a parallel magnetic field}

Magnetic field affects the unstable processes of the nucleation, suppressing or enhancing them, so that the morphology of deposit is drastically changed. In a magnetic field, electrochemical reaction induces the fluid motion by Lorentz force called MHD flow, which enhances mass transfer (MHD effect). At the same time, the MHD flow generates minute 
vortexes and convection cells called micro-MHD flows, which are the nonequilibrium fluctuations of MHD flow, often interacting with other nonequilibrium fluctuations, i.e., asymmetrical and symmetrical fluctuations accompanying nucleation; for 3D nucleation, the growth of symmetrical fluctuation is suppressed, and the sizes of 3D nuclei decrease (1st micro-MHD effect)(Fig. 4b). For 2D nucleation, asymmetrical fluctuations develop with secondary nodules (2nd micro-MHD effect)(Fig. 5b).

In Fig. 1, the magnetic flux density is applied in z-direction, and the current flows in ydirection, so that the resultant MHD main flow occurs in x-direction. In the boundary layer, micro-MHD flows arise from hydrodynamic and MHD interactions. The equations of the nonequilibrium fluctuations including micro MHD flows on the solution side under a parallel magnetic field have been established (Morimoto et al., in the course of submission-a). The equations are changed to the amplitude equations by Fourier transformation with respect to $x$ and y-directions. In view of the low electric conductivity and small representative length of electrochemical system, the effect of electromagnetic induction can be disregarded. For calculating the first and second micro-MHD effects, the amplitude equations are solved; the amplitude of the z-component of the velocity fluctuation $w$ is

$$
W^{0}(z, t)=A_{2}(t) z^{2} e^{-k z}
$$

and the amplitude of the z-component of the vorticity fluctuation $\omega_{z}$ is

$$
\Omega^{0}(z, t)=-i 4 T^{*}\left(\frac{B_{0}}{\rho v}\right)\left\langle\delta_{c}\right\rangle^{-3 / 4}\left(\frac{k}{k_{y}}\right) A_{2}(t) z e^{-k z}
$$

Then, at the electrode surface, the amplitude of the concentration fluctuation $c_{m}$ is expressed by

$$
\begin{aligned}
& \Theta^{0}(0, t)= \\
& -\frac{4 k^{3}\left(32 T^{*}\left\langle\delta_{c}\right\rangle^{1 / 4} k^{5}+z_{m} F \theta_{\infty}^{*} k_{y}^{2}\right)-i B_{x}^{*}\left\langle\delta_{c}\right\rangle^{-3 / 4} k_{x}\left(16 T^{*}\left\langle\delta_{c}\right\rangle^{1 / 4} k^{5}+5 z_{m} F \theta_{\infty}^{*} k_{y}^{2}\right)}{2 z_{m} F D_{m}\left\langle\delta_{c}\right\rangle k^{4} k_{y}^{2}\left(8 k^{3}+i B_{x}^{*}\left\langle\delta_{c}\right\rangle^{-3 / 4} k_{x}\right)} A_{2}(t)
\end{aligned}
$$

where $k_{x}$ and $k_{y}$ are the $\mathrm{x}$ - and y-components of the wave number $k$, respectively, and

$$
\begin{gathered}
T^{*} \equiv 1.6307 \mu_{0}\left(\gamma z_{m} F D_{m}\right)^{3 / 4} v^{3 / 2} \rho^{5 / 4} L^{1 / 4} \theta_{\infty}^{* 3 / 4} B_{0}^{-9 / 4} \\
B_{x}^{*}=1.6307\left(\gamma z_{m} F D_{m} \rho^{-1}\right)^{3 / 4}\left(\frac{v}{D_{m}}\right) v^{-3 / 2} L^{1 / 4}\left(\theta_{\infty}^{*} B_{0}\right)^{3 / 4}
\end{gathered}
$$

The amplitude of the concentration gradient fluctuation at the electrode surface is written by

$$
D \Theta^{0}(0, t)=\frac{8 T^{*}}{z_{m} F D_{m}\left\langle\delta_{c}\right\rangle^{3 / 4}}\left(\frac{k}{k_{y}}\right)^{2} A_{2}(t)
$$

where $D \equiv \partial / \partial z$, the coefficient $A_{2}(t)$ is an arbitrary function of time, and $\gamma$ is the cell constant of MHD electrode. $\mu_{0}$ is the magnetic permeability, $v$ is the kinematic viscosity, 
$\rho$ is the density, and $D_{m}$ is the diffusion coefficient. $B_{0}$ is the magnetic flux density, $L$ is the electrode length, and $\theta_{\infty}^{*}$ is the concentration difference between the bulk and surface. Equations (31) and (34) are connected to calculate $A_{2}(t)$ with the amplitudes of the fluctuations on the electrode side. In the following sections, the amplitudes of the symmetrical and asymmetrical fluctuations on the electrode side are derived.

\subsection{First micro-MHD effect}

In electrodeposition, hydrated metallic ions are traveling from the bulk to the electric double layer through the diffusion layer. At the double layer, dehydration first takes place, and adsorption follows at the Helmholtz layer. Transferring electrons, the adsorbed ions become adatoms, and some of them take part in nucleation. Other adatoms, according to the difference of surface energy, transfer along the electrode surface, settling as lattice atoms. Since the rate of electron transfer is sufficiently high, the mass transfer processes in the solution phase and the crystal phase become rate-determining steps, so that the electron transfer process is assumed in quasi-equilibrium state.

The mass balance of adatoms consists of the mass flux density of metallic ions from solution phase and the mass flux densities of adatoms by surface diffusion and incorporation to crystal lattices, i.e., $\vec{j}_{\text {flux }}^{s}, \vec{j}_{\text {surf }}^{s}$ and $\vec{j}_{\text {inc }}^{s}$, respectively.

$$
\frac{\partial}{\partial t} c_{a d}(x, y, t)^{s}+\nabla_{\perp} \cdot \vec{j}_{\text {surf }}^{s}=\vec{n} \cdot \vec{j}_{\text {flux }}^{s}-\vec{n} \cdot \vec{j}_{\text {inc }}^{s}
$$

where $c_{a d}(x, y, t)^{s}$ is the symmetrical fluctuation of the adatom concentration, $\vec{n}$ is the unit normal vector of the electrode surface, and $\nabla_{\perp} \equiv(\partial / \partial x, \partial / \partial y)$. Equation (35) is explicitly described as (Morimoto et al., in the course of submission-b)

$$
\frac{1}{\Omega_{m}} \frac{\partial}{\partial t} \zeta(x, y, t)^{s}=-\Omega_{m} \gamma^{*} \frac{D_{a d}}{R T} C_{a d}^{*} \nabla_{\perp}^{4} \zeta(x, y, t)^{s}+D_{m}\left\{\vec{n} \cdot \nabla c_{m}(x, y, z, t)^{s}\right\}
$$

where $\nabla_{\perp}^{4} \equiv \partial^{4} / \partial x^{4}+\partial^{4} / \partial y^{4} . \Omega_{m}$ is the molar volume, $\gamma^{*}$ is the surface energy, $D_{a d}$ is the surface diffusion coefficient, and $C_{a d}^{*}$ is the adatom concentration.

Fourier transformation of Eq. (36) allows us to derive the amplitude equation.

$$
\frac{1}{\Omega_{m}} \frac{\partial}{\partial t} Z^{0}(t)^{s}=-\Omega_{m} \gamma^{*} \frac{D_{a d}}{R T} C_{a d}^{*} k^{4} Z^{0}(t)^{s}+D_{m} D \Theta^{0}(0, t)^{s}
$$

Substituting for $D \Theta^{0}(0, t)^{s}$ from Eq. (34) in Eq. (37), we finally have the amplitude equation of $\zeta^{s}$

$$
\frac{1}{Z^{0}(t)^{s}} \frac{d}{d t} Z(t)^{s}=p
$$

Equation (38) is solved as

$$
Z^{0}(t)^{s}=Z^{0}(0)^{s} \exp (p t)
$$


where $p$ is the amplitude coefficient, i.e.,

$$
p=-\Omega_{m}^{2} \gamma^{*} \frac{D_{a d}}{R T} C_{a d}^{*} k^{4}+\alpha_{m M H D} \Omega_{m} D_{m} k\left\{L_{m}-\frac{\Omega_{m} \gamma^{*}}{R T}\left\langle C_{m}(x, y, 0, t)\right\rangle k^{2}\right\}
$$

where $\alpha_{m M H D}$ is the micro-MHD coefficient describing the effect of the micro-MHD flow on 3D nucleation, being expressed by

$$
\alpha_{m M H D} \equiv \frac{16 T^{*}\left\langle\delta_{c}\right\rangle^{1 / 4} k^{5}\left(8 k^{3}+i B_{x}^{*}\left\langle\delta_{c}\right\rangle^{-3 / 4} k_{x}\right)}{4 k^{3}\left(32 T^{*}\left\langle\delta_{c}\right\rangle^{1 / 4} k^{5}+z_{m} F \theta_{\infty}^{*} k_{y}^{2}\right)-i B_{x}^{*}\left\langle\delta_{c}\right\rangle^{-3 / 4} k_{x}\left(16 T^{*}\left\langle\delta_{c}\right\rangle^{1 / 4} k^{5}+5 z_{m} F \theta_{\infty}^{*} k_{y}^{2}\right)}
$$

As shown in Eq. (39c), $\alpha_{m M H D}$ is a complex number, acting as a spatial filter for the fluctuations, which controls the first micro-MHD effect, decreasing nucleus size. Actually, in the case of $\alpha_{m M H D}=1$, the amplitude coefficient $p$ is consistent with that in zero magnetic field (Aogaki et al., 1980). However, in general, due to the complex number of $\alpha_{m M H D}$, the amplitude coefficient $p$ also becomes complex number; Eq. (39a) is rewritten as

$$
Z^{0}(t)^{s}=Z^{0}(0)^{s} \exp (\operatorname{Re} p t) \exp (i \operatorname{Im} p t)
$$

where $\operatorname{Re} p$ and $\operatorname{Im} p$ denote the real and imaginary parts of $p$. In Eq. (40a), the part of $\exp (i \operatorname{Im} p t)$ expresses oscillation with time. However, because of the smallness of the $\operatorname{Im} p$, in comparison with the representative time of $3 \mathrm{D}$ nucleation, the period of the oscillation is quite long, so that such oscillation can be neglected, i.e.,

$$
Z^{0}(t)^{s}=Z^{0}(0)^{s} \exp (\operatorname{Re} p t)
$$

In Eq. (39b), as long as $L_{m}$ is positive $\operatorname{Re} p$ becomes positive for some wave number $k$, which leads to the unstable growth of 3D nuclei discussed in Section 2.2. The real part $\operatorname{Re} \alpha_{m M H D}$ is a spatial high-pass filter concerning the wave number of fluctuation. According to Eq. (40b), the component of fluctuation corresponding to the wave number region of $\operatorname{Re} p$ $>0$ can unstably develops with time. In this process, since $\operatorname{Re} \alpha_{m M H D}$ restricts such unstable growth of 3D nucleus with smaller wave number (larger nucleus size), first micro-MHD effect emerges.

$\mathrm{Z}^{0}(0)^{s}$ is the initial amplitude of the symmetrical surface fluctuation. In view of the thermal motion in atomic scale, assuming an isotropic white noise with normalization, we obtain the equation of the initial amplitude,

$$
\left|Z^{0}(0)^{s}\right|^{2}=\frac{X Y}{\pi} \frac{\left\langle\zeta_{c r}^{s 2}\right\rangle}{k_{\max }^{2}}
$$


where $\left\langle\zeta_{c r}^{s 2}\right\rangle$ is the mean square height of surface fluctuation in atomic scale at the initial state. $X$ and $Y$ are the $x$ - and $y$-lengths of the electrode, respectively. $k_{\max }$ is the upper limit of the wave number. Inserting Eq. (40a) into Eq. (37), and using Eq. (39b), we obtain

$$
D \Theta^{0}(0, t)^{s}=\alpha_{m M H D} k\left(L_{m}-\frac{\Omega_{m} \gamma^{*}}{R T}\left\langle C_{m}(x, y, 0, t)\right\rangle k^{2}\right) Z^{0}(0)^{s}
$$

By using Fourier inversion, $\operatorname{Re} D \Theta^{0}(0, t)^{s}$ is converted to $\left\{\partial c_{m}(x, y, z, t)^{s} / \partial z\right\}_{z=0}$, i.e.,

$$
\left\{\frac{\partial c_{m}(x, y, z, t)^{s}}{\partial z}\right\}_{z=0}=\frac{1}{2 \pi} \int_{-\infty}^{\infty} \int_{-\infty}^{\infty} \operatorname{Re} D \Theta^{0}(0, t)^{s} \exp \left[-i\left(k_{x} x+k_{y} y\right)\right] d k_{x} d k_{y}
$$

Then, the mass flux of the symmetrical fluctuation is given by

$$
j_{f l u x}(x, y, 0, t)^{s}=-D_{m}\left\{\frac{\partial c_{m}(x, y, z, t)^{s}}{\partial z}\right\}_{z=0}
$$

The effective surface heights of the 3D nuclei are also calculated in the following,

$$
\zeta(x, y, t)^{s}=\frac{1}{\Omega_{m}} \int_{0}^{t} j_{f l u x}(x, y, 0, t)^{s} d t
$$

a

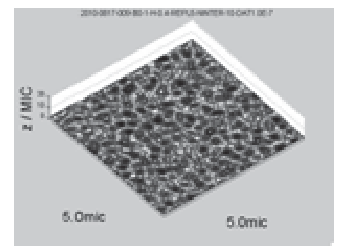

a1

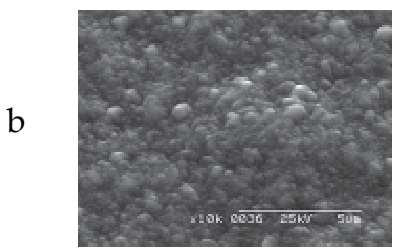

b1

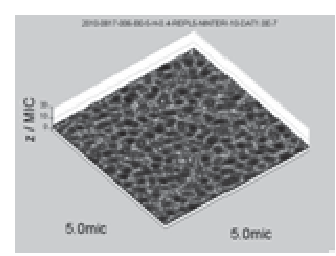

a2

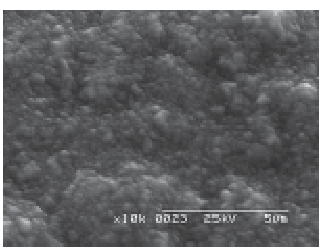

b2

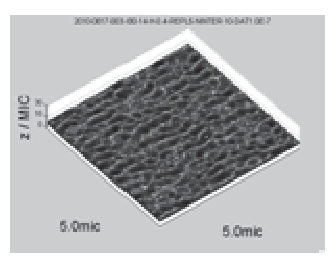

a3

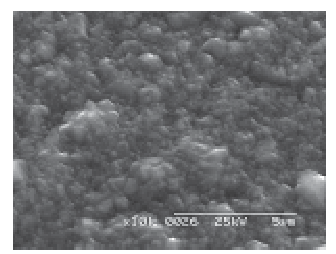

b3

Fig. 13. First micro-MHD effect. a; calculation of the morphology of copper 3D nuclei. a, calculation: a1, $B_{0}=0 \mathrm{~T}$; a2, $B_{0}=5 \mathrm{~T} ; \mathrm{a} 3, B_{0}=14 \mathrm{~T}$. b, SEM image: b1, $B_{0}=0 \mathrm{~T} ; \mathrm{b} 2, B_{0}=5$ $\mathrm{T} ; \mathrm{b} 3, B_{0}=14 \mathrm{~T}$ (Morimoto et al., in the course of submission-b). 


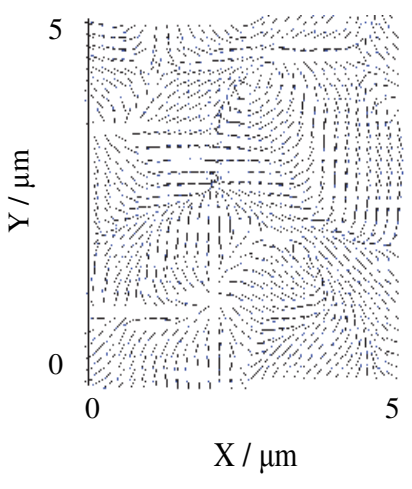

a

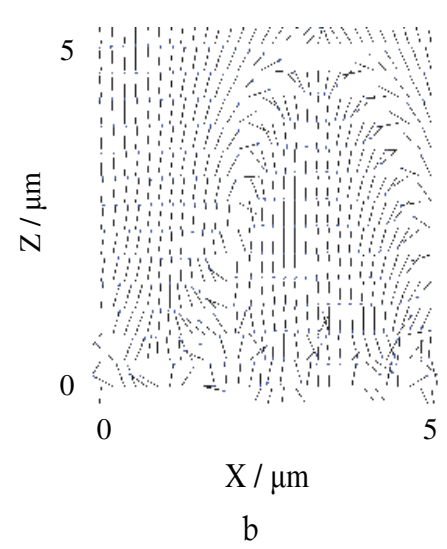

$\mathrm{b}$

Fig. 14. Horizontal and cross-sectional distributions of micro-MHD flow. a, horizontal distribution; $b$, cross-sectional distribution. $B_{0}=5 \mathrm{~T}$ (Morimoto et al., in the course of submission-b).

In Fig. 13, the theoretical calculation and experimental result of first micro-MHD effect are exhibited; as magnetic flux density increases, the size of 3D nucleus decreases. In Fig. 14, the micro-MHD flows corresponding to the 3D nucleation are exhibited.

\subsection{Second micro-MHD effect}

\subsubsection{Instability equation}

Due to large scale of length $(\approx 100 \mu \mathrm{m})$, the asymmetrical fluctuations controlling 2D nucleation result not from the nucleation process on the electrode surface but from the electrochemical reaction process in the electrical double layer and the micro-MHD flow in the convective diffusion layer; since the term with higher power of wave number is neglected, the equation corresponding to Eq. (37) is simply expressed by

$$
\frac{1}{\Omega_{m}} \frac{\partial}{\partial t} Z^{0}(t)^{s}=D_{m} D \Theta^{0}(0, t)^{s}
$$

At the same time, from Eqs. (5), (19), (26) and (28), the following relationship is finally derived as

$$
\Theta^{0}(0, t)^{a}=-\frac{z_{m} F}{R T}\left(\frac{\partial\left\langle\Phi_{1}\right\rangle}{\partial\left\langle\Phi_{2}\right\rangle}\right)_{\mu} L_{\phi}^{a} C_{m}^{*}(0, t) Z^{0}(t)^{a}
$$

Since 2D nucleation is controlled by micro-MHD flow, not the surface height fluctuation but the concentration fluctuation determines the Gaussian power spectrum. The amplitude of the concentration fluctuation is thus expressed by

$$
\begin{gathered}
\left|\Theta^{0}(0, t)^{a}\right|^{2}=\frac{X Y}{\pi} \theta_{\infty}^{* 2} a^{+2} \exp \left(-a^{+2} k^{2}\right) \\
a^{+}=\frac{\left\langle\delta_{c}\right\rangle}{m}
\end{gathered}
$$


where $a^{+}$is the autocorrelation distance of the fluctuations, which is defined by the average thickness of the convective-diffusion layer $\left\langle\delta_{c}\right\rangle$ and the number of vortexes $m$ vertically standing in a line between the electrode and the diffusion layer boundary, i.e., the $a^{+}$is equal to the average size of the vortex.

Substituting Eq. (47) into Eq. (46), we obtain the evolution equation of the asymmetrical fluctuation.

$$
\frac{\partial \Theta^{0}(0, t)^{a}}{\partial t}=A_{\theta} D \Theta^{0}(0, t)^{a}
$$

where

$$
A_{\theta} \equiv \frac{z_{m} F D_{m} \Omega_{m} C_{m}^{*}(0, t)\left\langle\Phi_{2}\right\rangle}{\lambda R T}\left(\frac{\partial\left\langle\Phi_{1}\right\rangle}{\partial\left\langle\Phi_{2}\right\rangle}\right)_{\mu}
$$

The unstable condition is therefore

$$
A_{\theta}>0
$$

Namely, with Eq. (49c), the same instability analysis as discussed in Section 2.3 is also possible. Substituting for $\Theta^{0}(0, t)^{a}$ and $D \Theta^{0}(0, t)^{a}$ from Eqs. (31) and (34) in Eq. (49a), we obtain the equation of the coefficient $A_{2}(t)$ as follows,

$$
\frac{d A_{2}(t)^{a}}{d t}=h\left(\left\langle\delta_{c}\right\rangle\right) A_{2}(t)^{a}
$$

where

$$
h\left(\left\langle\delta_{c}\right\rangle\right)=-16 T^{*} A_{\theta} k^{6} h\left(\left\langle\delta_{c}\right\rangle\right)^{+}
$$

The explicit form of $h\left(\left\langle\delta_{c}\right\rangle\right)^{+}$is given by

$$
h\left(\left\langle\delta_{c}\right\rangle\right)^{+} \equiv \frac{\left\langle\delta_{c}\right\rangle^{1 / 4}\left(8 k^{3}+i B_{x}^{*}\left\langle\delta_{c}\right\rangle^{-3 / 4} k_{x}\right)}{4 k^{3}\left(32 T^{*}\left\langle\delta_{c}\right\rangle^{1 / 4} k^{5}+z_{m} F \theta_{\infty}^{*} k_{y}^{2}\right)-i B_{x}^{*}\left\langle\delta_{c}\right\rangle^{-3 / 4} k_{x}\left(16 T^{*}\left\langle\delta_{c}\right\rangle^{1 / 4} k^{5}+5 z_{m} F \theta_{\infty}^{*} k_{y}^{2}\right)} \text { (50c) }
$$

\subsubsection{Calculation of the average thickness of diffusion layer $\left\langle\delta_{c}\right\rangle$}

To calculate the coefficient $A_{2}(t)$ in Eq. (50a), as shown in Eq. (48b), it is necessary to determine the value of $\left\langle\delta_{c}\right\rangle$. After long-term deposition, it is thought that the asymmetrical concentration fluctuation has already developed to the maximum point, so that the secondary nodule formation fulfills the following condition concerning the mean square value of the asymmetrical concentration fluctuation over the electrode surface. 


$$
\frac{d}{d t}\left\langle\left|c_{m}(x, y, 0, t)^{a}\right|^{2}\right\rangle \approx 0
$$

Here, the Rayleigh theorem suggests the relationship

$$
\left\langle\left|c_{m}(x, y, 0, t)^{a}\right|^{2}\right\rangle=\frac{1}{X Y} \int_{-\infty}^{\infty} \int_{-\infty}^{\infty}\left|\Theta^{0}(0, t)^{a}\right|^{2} d k_{x} d k_{y}
$$

From Eq. (51b), more generally, it can be said that the mean square value of the fluctuation is calculated by the integration of that of the amplitude. Although the concentration fluctuation has already grown up to its ultimate state, the gradient of the fluctuation can still develop with new components of the fluctuation; this inevitable development of the microMHD convection leads to the decrease of the convective-diffusion layer thickness, promoting mass transfer process (Fig. 5). In terms of the mean square values of the concentration fluctuation and its gradient, the average thickness of the convective-diffusion layer is defined by

$$
\left(\frac{\left\langle\delta_{c}\right\rangle}{m}\right)^{2} \equiv \frac{\left\langle\left|c_{m}(x, y, 0, t)^{a}\right|^{2}\right\rangle}{\left\langle\left|\left(\frac{\partial c_{m}(x, y, z, t)}{\partial z}\right)_{z=0}\right|^{2}\right\rangle}
$$

From Eq. (48b), the term on the left hand side in Eq. (52) is equal to the square value of the autocorrelation distance of the fluctuation. By differentiating Eq. (52) with respect to time, the following nonlinear evolution equation is derived.

$$
\frac{d\left\langle\delta_{c}\right\rangle}{d t}=-f\left(\left\langle\delta_{c}\right\rangle\right)\left\langle\delta_{c}\right\rangle
$$

where

$$
\left.\left.f\left(\left\langle\delta_{c}\right\rangle\right) \equiv \frac{\frac{d}{d t}\left\langle\left(\frac{\partial c_{m}(x, y, z, t)^{a}}{\partial z}\right)_{z=0} \mid{ }^{2}\right\rangle}{2\left\langle\left|\left(\frac{\partial c_{m}(x, y, z, t)^{a}}{\partial z}\right)_{z=0}\right|\right.}\right|^{2}\right\rangle \frac{\frac{d}{d t}\left\langle\left|c_{m}(x, y, 0, t)^{a}\right|^{2}\right\rangle}{2\left\langle\left|c_{m}(x, y, 0, t)^{a}\right|^{2}\right\rangle}
$$

After solving Eq. (53a), the average current density is calculated by

$$
\left\langle j_{z}^{a}\right\rangle=-z_{m} F D_{m} \theta_{\infty}^{*} /\left\langle\delta_{c}\right\rangle
$$

In the case of copper deposition from sulfuric acid solution, the condition $\left(\partial\left\langle\Phi_{1}\right\rangle / \partial\left\langle\Phi_{2}\right\rangle\right)_{\mu}>0$ are generally fulfilled (Aogaki et al., 2010), so that from Eq. (49c), a positive diffuse layer overpotential $\left\langle\Phi_{2}\right\rangle>0$ is required for secondary nodule formation. 
However, since copper deposition is a cathodic reaction, the diffuse layer overpotential is usually supposed negative, of which contradiction is, as discussed in Section 2.3, solved by the adsorption of cation such as proton in the double layer, where the positive charges of protons adsorbed on the Helmholtz layer shift the overpotential to positive side. Such discussion has been validated in Fig. 15 by the scanning electron microscope (SEM) images for the copper depositions with and without the adsorption of protons, i.e., the secondary nodules can be seen only in the case of proton adsorption.

Theoretical current-time curve was calculated by means of Eqs. (53a) and (53b). Figure 16 exhibits the comparison between theoretical calculation and experimental result of currenttime curves for the copper deposition. Both curves are in good agreement, quite slowly increasing up to a steady state. From the calculation, it is estimated that the value of $\left\langle\Phi_{2}\right\rangle$ is small, i.e., the order of $1 \mathrm{mV}$. To ascertain the above discussion, based on the theory, we calculated the surface morphology of secondary nodules and the velocity distribution of the micro-MHD flows. Figure 17 represents the surface morphology, and Fig. 18 represents the horizontal and vertical velocity distributions of the corresponding micro-MHD vortexes.

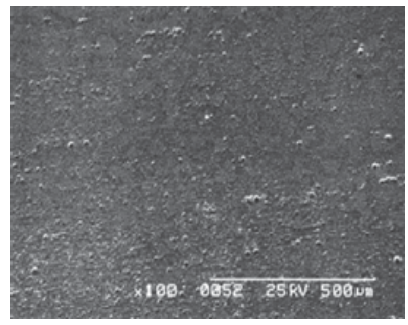

a

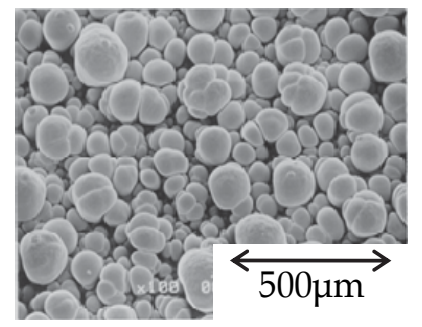

b

Fig. 15. SEM photos of secondary nodules. a, without the adsorption of $\mathrm{H}^{+}$ions; $b$, with the adsorption of $\mathrm{H}^{+}$ions (2nd micro-MHD effect) (Aogaki et al., 2010).

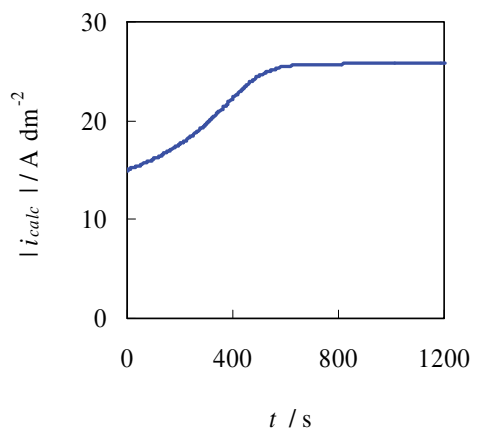

a

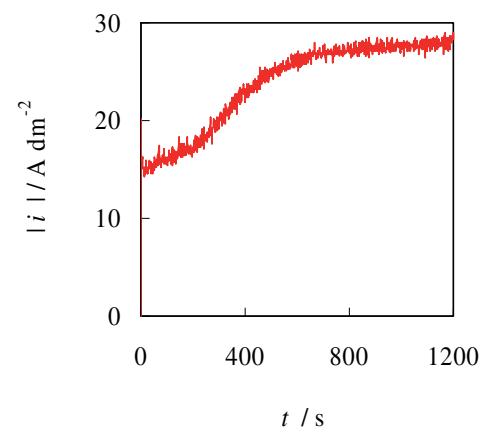

b

Fig. 16. Current-time curves for secondary nodule formation in copper deposition up to 1200 s. a; calculation, b; experimental result. Applied overpotential, -0.4 V; bulk concentration, $300 \mathrm{~mol} \mathrm{~m}^{-3}$. $B=5 \mathrm{~T}$ (Aogaki et al., 2010). 


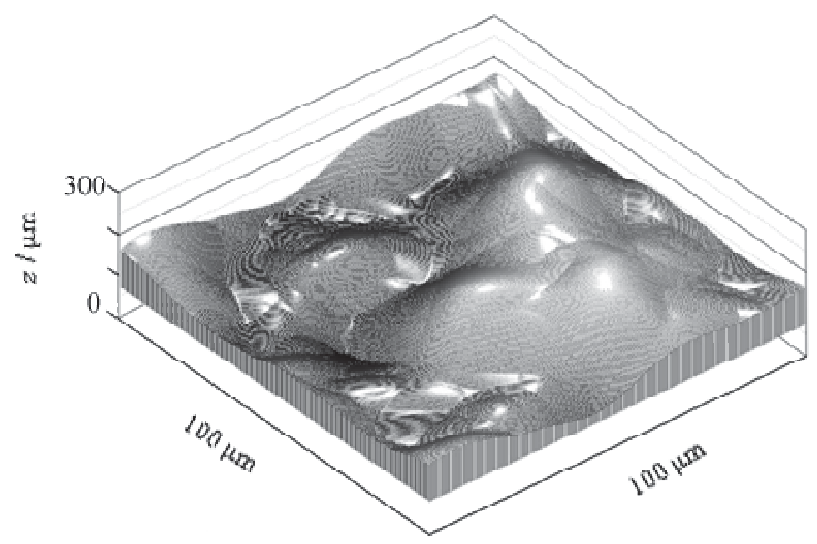

Fig. 17. Calculated surface morphology of copper secondary nodules. $B=5 \mathrm{~T}$. Deposition time is $1000 \mathrm{~s}$, and concentration is $300 \mathrm{~mol} \mathrm{~m}^{-3}$. Nucleation takes place at the interval of $10 \mathrm{~s}$ (Aogaki et al., 2010).

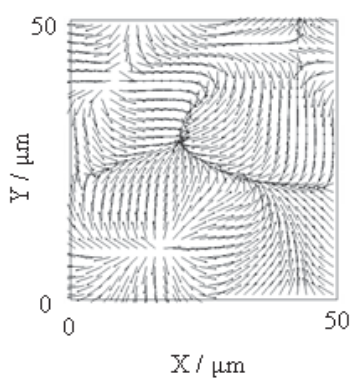

a

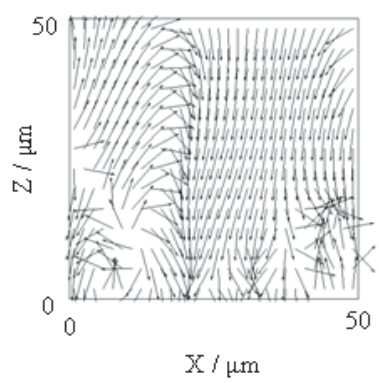

b

Fig. 18. 2nd micro-MHD flows in the formation of copper secondary nodules at $5 \mathrm{~T}$.

$\mathrm{a}$, horizontal motion at $z=30 \mu \mathrm{m} ; \mathrm{b}$, cross-sectional motion at $y=30 \mu \mathrm{m}$, where the $x y$-plane is taken as an electrode surface, and the $z$ coordinate is used for deposition height.

\section{Chirality appearance in a vertical magnetic field}

In electrodeposition under a vertical magnetic field, as discussed in 1. Introduction, it is thought that instead of vortexes with chiral symmetry, vortexes having chirality emerge. The best way to make clear the process of the evolution of the chirality is to pursue how chiral symmetry is broken down from a perfectly symmetrical state. Instead of vertical MHD flow, as shown in Fig. 19, a more simple case is taken where an electrochemical system is rotating under a vertical uniform magnetic field, and also it is assumed that current density is uniform and the current lines are vertical everywhere. It should be noted that the whole system including the electrodes and solution rotates all in one body. 


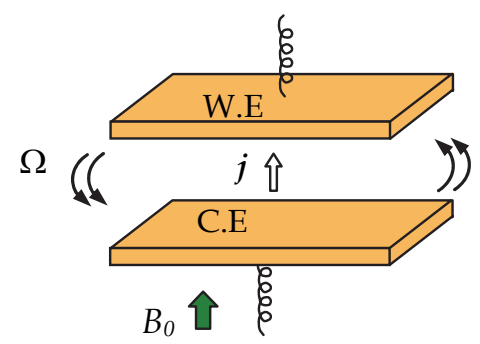

Fig. 19. Schematic of a rotating electrolysis system. W.E, working electrode; C.E., counter electrode; $\Omega$, angular velocity; $B_{0}$, magnetic flux density (Aogaki et al., 2009d).

After starting electrodeposition, nonequilibrium fluctuations are introduced; asymmetrical fluctuations for 2D nucleation and symmetrical fluctuations for 3D nucleation. Then, the evolution of the fluctuations is calculated within MHD framework. In this case, whole system is rotating in an angular velocity $\Omega(>0)$ clockwise when seeing the solution from electrode side. The rotating axis of this system is perpendicular, and the magnetic flux density $B_{0}(>0)$ is upward applied vertically to the electrodes. The nonequilibrium fluctuation equations to describe the micro-MHD flows and the concentration fluctuation are first derived, which are then transformed to the amplitude equations by Fourier transformation. The amplitude equations are solved under the boundary conditions concerning rigid and free surfaces. For a rigid surface under a downward flow $w_{r}$ (z-component of the velocity) $<0$, at the electrode surface, the amplitude of the concentration gradient fluctuation is expressed by

$$
D \Theta_{r}^{0}(0, t)=\frac{12 a_{1}}{5 z_{m} F D_{m} S}
$$

The amplitude of the concentration fluctuation at the electrode surface is

$$
\Theta_{r}^{0}(0, t)=-\frac{384 k^{5}+9 z_{m} F D_{m} S T_{r} R^{*}}{160 z_{m} F D_{m} S k^{6}} a_{1}
$$

For a free surface under an upward flow $w_{f}$ (z-component of the velocity) $>0$, at the electrode surface, the amplitude of the concentration gradient fluctuation is

$$
D \Theta_{f}^{0}(0, t)=\frac{12 k a_{0}}{5 z_{m} F D_{m} S}
$$

The amplitude of the concentration fluctuation at the electrode surface is

$$
\Theta_{f}^{0}(0, t)=-\frac{96 k^{5}-5 z_{m} F D_{m} S T_{f} R^{*}}{40 z_{m} F D_{m} S k^{5}} a_{0}
$$

where

$$
S \equiv \frac{B_{0}}{\rho v}
$$




$$
\mathrm{T}_{j} \equiv \pm \frac{2 \Omega}{v} \text { for } \mathrm{j}=\operatorname{ror} \mathrm{f}
$$

where as will be discussed later, subscripts ' $r$ ' and ' $\mathrm{f}$ ' imply rigid and free surfaces, respectively. The signs ' + ' and '-' are taken for the clockwise and counterclockwise rotations corresponding to the signs of the z-component of the vorticity $\omega_{z j}$, respectively when seeing the solution from electrode side. Then, it follows that

$$
R^{*} \equiv \frac{L_{m}}{D_{m}}
$$

$a_{0}$ and $a_{1}$ are arbitrary coefficients.

\subsection{Formation of micro-mystery circle}

\subsubsection{Occurrence of chirality}

As discussed initially, micro-mystery circle is treated as a 2D nucleus arising from asymmetrical fluctuations. For asymmetrical fluctuation, as discussed in the former sections, due to much larger scale of length of asymmetrical fluctuation than that of symmetrical fluctuation, not nucleation process on the electrode but electrochemical process in the electric double layer controls the total reaction. Therefore, the same amplitude equations as Eqs. (49a) and (49b) are derived. The unstable condition for the micro-mystery circle formation is also the same as Eq. (49c), which means that the micro-mystery circle formation in a vertical magnetic field follows the same unstable condition as the secondary nodule formation in a parallel magnetic field. Namely, when proton adsorption occurs, micromystery circle is self-organized.

However, the equations of the coefficients $a_{0}$ and $a_{1}$ are different from the same kind of equation Eq. (50a). Substituting for $D \Theta_{r}^{0}(0, t)$ and $\Theta_{r}^{0}(0, t)$ from Eqs. (55a) and (55b) in Eq. (49a), we have the coefficient equation for a rigid surface, i.e.,

$$
\frac{d a_{1}^{a}}{d t}=-A_{\theta} f_{r}^{a}(k) a_{1}^{a}
$$

and

$$
f_{r}^{a}(k)=\frac{128 k^{6}}{128 k^{5}+3 \alpha_{r}^{a^{*}}}
$$

where

$$
\alpha_{r}^{a^{*}}=z_{m} F D_{m} S^{a} T_{r}^{a} R^{*}
$$

$S^{a}, T_{r}^{a}$ and $R^{*}$ are the parameters expressing magnetic field, rotation and mass transfer, respectively. Here, $R^{*}$ is positive in Eq. (59). To avoid the singular point of $k$ in Eq. (60b), the condition

$$
S^{a} T_{r}^{a}>0
$$


is required. For the external magnetic flux density defined by $B_{0}>0, S^{a}$ is also positive in Eq. (57). In view of Eq. (58), $T_{r}^{a}$ must be thus positive, being defined by

$$
\mathrm{T}_{r}^{a}=+\frac{2 \Omega}{v}
$$

Since $\Omega$ is the angular velocity of the rotation of the system, and assumed to be positive, i.e., clockwise, the + sign indicates that the micro-MHD flow on a rigid surface rotates clockwise, i.e., in the same direction as that of the system, i.e., $w_{r}^{a}<0$ and $\omega_{z r}^{a}>0$.

For a free surface, substituting for $D \Theta_{f}^{0}(0, t)$ and $\Theta_{f}^{0}(0, t)$ from Eqs. (56a) and (56b) in Eq. (49a), we get

$$
\frac{d a_{0}^{a}}{d t}=-A_{\theta} f_{f}^{a}(k) a_{0}^{a}
$$

where

$$
f_{f}^{a}(k)=\frac{96 k^{6}}{96 k^{5}-5 \alpha_{f}^{a^{*}}}
$$

where

$$
\alpha_{f}^{a^{*}}=z_{m} F D_{m} S^{a} T_{f}^{a} R^{*}
$$

Since $R^{*}$ is, as discussed above, positive. In order to avoid the singular point of $k$ in Eq. (63b), the condition

$$
S^{a} T_{f}^{a}<0
$$

is required. Since $S^{a}$ is positive,

$$
\mathrm{T}_{f}^{a}=-\frac{2 \Omega}{v}<0
$$

must be fulfilled. In comparison with Eq. (62), Eq. (65) indicates that the micro-MHD vortexes on a free surface rotates in the direction reverse to that of the vortexes on a rigid surface, i.e., $w_{f}^{a}>0$ and $\omega_{z f}^{a}<0$.

\subsubsection{Formation of micro-mystery circle}

To treat the two independent self-organization processes concerning rigid and free surfaces at one time, it is necessary to describe the amplitudes in complex numbers. In Fourier inversion, utilizing the characteristic nature of complex Fourier transform, where even and odd functions are simultaneously transformed in the real and imaginary parts, we treat the rigid surface and free surface components as even and odd functions, respectively.

In consideration of the stochastic process in multiple nucleus formation, the following unit complex number is introduced. 


$$
R_{d}^{a}=\cos \theta_{\text {rand }}^{a}+i \sin \theta_{\text {rand }}^{a}
$$

where $\theta_{\text {rand }}^{a}$ is the uniform random number between 0 and $2 \pi$, which are assigned to all grid points defined in an electrolyte solution. From the amplitudes of the gradients of the concentration fluctuations, the actual concentration gradients are calculated by Fourier inversion as follows,

$$
\left\{\frac{\partial c_{m j}(x, y, z, t)}{\partial z}\right\}_{z=0}=\frac{1}{2 \pi} \int_{-\infty}^{\infty} \int_{-\infty}^{\infty} D \Theta_{j}^{0}(z, t)^{a} \exp \left[-i\left(k_{x} x+k_{y} y\right)\right] d k_{x} d k_{y} \text { for } \mathrm{j}=\mathrm{ror} \mathrm{f}
$$

where

$$
D \Theta_{r}^{0}(0, t)^{a}=\frac{12 \beta_{1}^{a} f_{r}^{a}(k) a^{+}}{5 z_{m} F D_{m} S_{r}^{a}} \exp \left(-\frac{a^{+2} k^{2}}{2}\right) R_{d}^{a}
$$

and

$$
\beta_{1}^{a}=\frac{5}{12} \alpha_{r}^{a}\left(\frac{X Y}{\pi}\right)^{1 / 2} z_{m} F D_{m} \theta_{\infty}^{*} S_{r}^{a}
$$

Then, we have

$$
D \Theta_{f}^{0}(0, t)^{a}=\frac{12 \beta_{0}^{a} f_{f}^{a}(k) a^{+}}{5 z_{m} F D_{m} S_{f}^{a}} \exp \left(-\frac{a^{+2} k^{2}}{2}\right) R_{d}^{a}
$$

where

$$
\beta_{0}^{a}=\frac{5}{12} \alpha_{f}^{a}\left(\frac{X Y}{\pi}\right)^{1 / 2} z_{m} F D_{m} \theta_{\infty}^{*} S_{f}^{a}
$$

As discussed initially, to automatically determine the rigid and free areas, the signs of the z-components of the velocity $w_{j}^{a}$ and vorticity $\omega_{z j}^{a}$ for $\mathrm{j}=\mathrm{r}$ or $\mathrm{f}$ are important. The actual z-components of velocity and vorticity fluctuations are calculated by the Fourier inversions. For a clockwise rotating system, under the conditions $w_{r}^{a}<0$ and $\omega_{z r}^{a}>0$, the current density on the rigid surface is calculated by

$$
j_{z r}(x, y, 0, t)^{a}=-z_{m} F D_{m}\left\{\frac{\partial c_{m r}(x, y, z, t)}{\partial z}\right\}_{z=0}
$$

Under the conditions $w_{f}^{a}>0$ and $\omega_{z f}^{a}<0$, the current density on the free surface is given by

$$
j_{z f}(x, y, 0, t)^{a}=-z_{m} F D_{m}\left\{\frac{\partial c_{m f}(x, y, z, t)}{\partial z}\right\}_{z=0}
$$


The total current density $j_{z}(x, y, 0, t)^{a}$ is thus expressed by

$$
j_{z}(x, y, 0, t)^{a}=j_{z r}(x, y, 0, t)^{a}+j_{z_{f}}(x, y, 0, t)^{a}
$$

As a result, the surface morphology of the 2D nucleation is effectively calculated by

$$
\zeta(x, y, t)^{a}=-\frac{\Omega_{m}}{z_{m} F} \int_{0}^{t} j_{z}(x, y, 0, t)^{a} d t
$$

In this calculation, only $1 / 4$ th of the total fluctuations is used at one time. This inadequacy is solved by multiple nucleus formation randomly occurring in time axis.

In Fig. 20a, an example of the theoretical calculation is exhibited. Owing to multiple random nucleus formation, though nucleation takes place all over the surface, four regular micromystery circles emerge. Figure 20b represents the 3D SEM image of a micro-mystery circle formed in copper deposition, which was observed in a rotating electrode system under a vertical magnetic field.

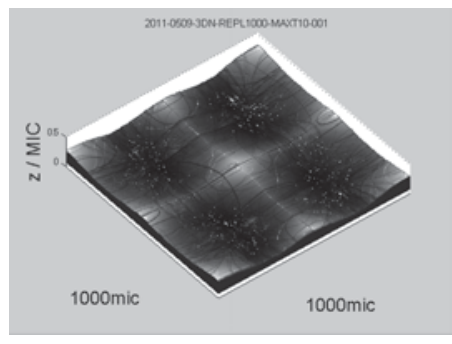

a

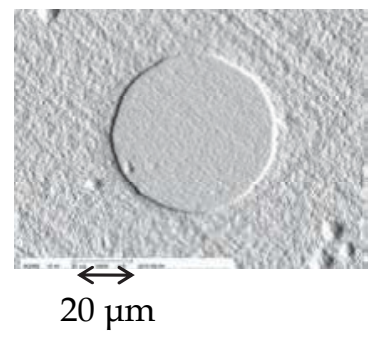

$\mathrm{b}$

Fig. 20. Morphology of micro-mystery circle. a, theoretical calculated morphology;

b, SEM image of copper micro-mystery circle in copper deposition of the rotating system.

\subsection{Formation of nano-mystery circle}

As have been discussed in last section, at the boundary on a free surface of $2 \mathrm{D}$ nucleus covered with ionic vacancies, the frictionless rotation of vortex reverse to the rotating system yields a concentric circular pattern of micro-mystery circle, whereas at the boundary on the rigid surface, due to friction, the solution is kept stationary. 3D nucleus formation on a rigid surface of $2 \mathrm{D}$ nucleus also receives the same effects as $2 \mathrm{D}$ nuclei, i.e., the precession to micro-MHD flows and the selection of the direction of rotation. However, there are some differences; due to small scale of length, i.e., low Reynolds number, the effective viscosity that vortexes feel becomes too high to rotate, so that rotations themselves become impossible. To solve this problem, a new effect of ionic vacancy to decrease viscosity is required.

Considering that ionic vacancies are easy to coalesce from a nature of bubble, we propose a new effect called 'micro-hydroplaning'. Due to coalescence, ionic vacancies are supposed to act like a creamy lubricant. Let us imagine a coffee surface with cream, where due to the difference of viscosity, without mixing, the cream forms white stripes surrounding coffee vortexes. If these stripes are replaced with those of ionic vacancies, just like a wheel in the 
well-known hydroplaning effect, a micro-vortex surrounded by the stripes of ionic vacancies can rotate freely. This phenomenon effectively decreases kinematic viscosity. Since ionic vacancies gather in an upward flow of a 3D nucleus, according to this effect, the local kinematic viscosity in the upward flow drastically decreases for rotation. As a result, in the downward flow without ionic vacancy, due to effectively high viscosity, the rotation itself is prohibited, remaining stationary, whereas in the upward flow containing the stripes of ionic viscosity, owing to effectively low viscosity, a vortex can rotate. In the same way as that of the micro-mystery circle formation, but quite different in size, a concentric circular pattern called nano-mystery circle with chirality is thus formed. At the center of the nanomystery circle, a screw dislocation with chirality emerges. In Fig. 21a, the theoretical calculation of nano-mystery circle is represented. In spite of random multiple nucleus formation, nano-mystery circles can be seen. Figure $21 \mathrm{~b}$ shows the SEM image of a copper deposit, where many shallow dimples in the same shape are observed.

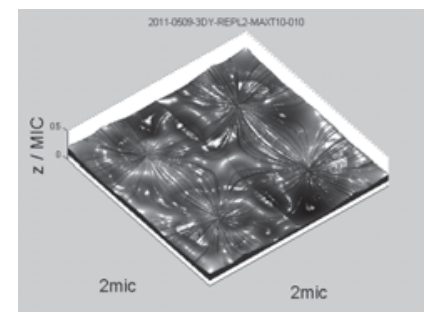

a

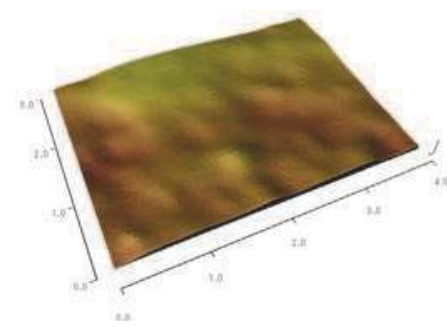

b

Fig. 21. Morphology of nano-mystery circle. a, theoretical calculation ( $2 \mu \mathrm{m}$ in full scale); $\mathrm{b}$, SEM image ofcopper nano-mystery circle in copper deposition of the rotating system (4 $\mu \mathrm{m}$ in full scale).

\section{Conclusion}

In a parallel magnetic field, usually an electrode system called MHD electrode is used, which consists of a rectangular channel with two open ends (Fig. 1). Arriving through the inlet, the solution moves along the electrode surface, and leaves out of the outlet. As a result, generated vacancies are continuously swept away from the electrode surface. This is the reason why the vacancy does not affect the deposition process in a parallel magnetic field.

\section{References}

Aogaki, R., Fueki, K., \& Mukaibo, T. (1975). Application of Magnetohydrodynamic Effect to the Analysis of Electrochemical Reactions -2. Diffusion Process in MHD Forced Flow of Electrolyte Solutions. DENKI KAGAKU (presently Electrochemistry), Vol. 43, No. 9, (August 1975), pp. 509-514, ISSN 1344-3542

Aogaki, R., Kitazawa, K., Kose, Y., \& Fueki, K. (1980). Theory of Powdered Crystal Formation in Electrocrystallization -Occurrence of Morphological Instability at the Electrode Surface. Electrochimica Acta, Vol. 25, No. 7, (July 1980), pp. 965-972, ISSN 0013-4686 
Aogaki, R. (1995). Instability of Nonequilibrium Fluctuation in Electrochemical Nucleation I. Occurrence of Instability. Journal of Chemical Physics, Vol. 103, No. 19, (November 1995), pp. 8602-8615, ISSN 0021-9606

Aogaki, R. (2001). Magnetic Field Effects in Electrochemistry. Magnetohydrodynamics, Vol. 37, No. 1/2, (August 2001), pp. 143-150, ISSN 0024-998X

Aogaki, R., Morimoto, R., Sugiyama, A., \& Asanuma, M. (2008a). Self-organization of Copper Secondary Nodules by the Second Micro-MHD Effect. ECS Transactions, Vol. 13, No. 16, (December 2008), pp. 15-24, ISSN 1938-6737

Aogaki, R. (2008b). Theory of Stable Formation of Ionic Vacancy in a Liquid Solution. Electrochemistry, Vol. 76, No. 7, (June 2008), pp. 458-465, ISSN 1344-3542

Aogaki, R., Morimoto, R., Sugiyama, A., \& Asanuma, M. (2009a). Double Layer Mechanism in Self-organization of Copper Secondary Nodules under a Parallel Magnetic Field. Magnetohydrodynamics, Vol. 45, No. 2, (April 2009), pp. 245-252, ISSN 0024-998X

Aogaki, R., Miura, M., \& Oshikiri, Y. (2009b). Origin of Nanobubble-Formation of Stable Vacancy in Electrolyte Solution. ECS Transactions, Vol. 16, No. 25, (May 2009), pp. 181-189, ISSN 1938-6737

Aogaki, R., Morimoto, R., Sugiyama, A., \& Asanuma, M. (2009c). Origin of chirality in magnetoelectrodeposition, Proceedings of 6th International Conference Electromagnetic Processing of Materials, pp. 439-442, ISBN 978-3-936104-65-3, Dresden, Germany, October 2009

Aogaki, R., Morimoto, R., \& Asanuma, M. (2010). Nonequilibrium Fluctuations in MicroMHD Effects on Electrodeposition. Journal of Magnetism and Magnetic Materials, Vol. 322, No. 9/12, (June 2010), pp. 1664-1668, ISSN 0304-8853

Mogi, I., \& Watanabe, K. (2005). Chirality of magnetoelectropolymerized polyaniline electrodes. Japan Journal of Applied Physics, Vol. 44, No. 5, (January 2005), pp. L199L201, ISSN 0021-4922

Mogi, I. (2008). Chiral Electrode Behaviors of Magnetoelecrodeposited Ag films for Amino Acids. ECS Transactions, Vol. 13, No. 16, (December 2008), pp. 45-52, ISSN 19386737

Sugiyama, A., Morimoto, R., Hashiride, M., Nagai, Y., \& Aogaki, R. (2004). Application of Vertical Micro-disk MHD electrode to the Analysis of Heterogeneous Magnetoconvection. Electrochimica Acta, Vol. 49, No. 28, (November 2004), pp. 5115-5124, ISSN 0013-4686

Morimoto, R., Sugiyama, A., \& Aogaki, R. (2004). Nano-scale Crystal Formation in Copper Magneto-electrodeposition Under Parallel Magnetic Fields. Electrochemistry, Vol. 72, No. 6, (May 2004), pp. 421-423, ISSN 1344-3542

Morimoto, R., Aogaki, R., Sugiyama, A., \& Asanuma, M. (n.d-a). Micro-MHD Effects in a Parallel Magnetic Fields -1. Equations of Micro-MHD Flows. in the course of submission to J. Chem. Phys.

Morimoto, R., Aogaki, R., Sugiyama, A., \& Asanuma, M. (n.d-b). Micro-MHD Effects in a Parallel Magnetic Fields -2. 1 ${ }^{\text {st }}$ Micro-MHD Effect: Suppression of Three Dimensional (3D) Nucleation. in the course of submission to J. Chem. Phys. 


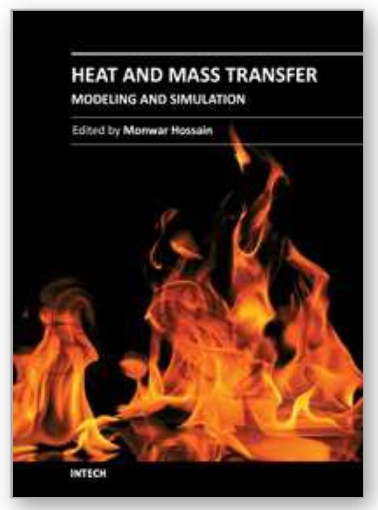

\author{
Heat and Mass Transfer - Modeling and Simulation \\ Edited by Prof. Md Monwar Hossain
}

ISBN 978-953-307-604-1

Hard cover, 216 pages

Publisher InTech

Published online 22, September, 2011

Published in print edition September, 2011

This book covers a number of topics in heat and mass transfer processes for a variety of industrial applications. The research papers provide advances in knowledge and design guidelines in terms of theory, mathematical modeling and experimental findings in multiple research areas relevant to many industrial processes and related equipment design. The design of equipment includes air heaters, cooling towers, chemical system vaporization, high temperature polymerization and hydrogen production by steam reforming. Nine chapters of the book will serve as an important reference for scientists and academics working in the research areas mentioned above, especially in the aspects of heat and mass transfer, analytical/numerical solutions and optimization of the processes.

\title{
How to reference
}

In order to correctly reference this scholarly work, feel free to copy and paste the following:

Ryoichi Aogaki and Ryoichi Morimoto (2011). Nonequilibrium Fluctuations in Micro-MHD Effects on Electrodeposition, Heat and Mass Transfer - Modeling and Simulation, Prof. Md Monwar Hossain (Ed.), ISBN: 978-953-307-604-1, InTech, Available from: http://www.intechopen.com/books/heat-and-mass-transfermodeling-and-simulation/nonequilibrium-fluctuations-in-micro-mhd-effects-on-electrodeposition

\section{INTECH}

open science | open minds

\section{InTech Europe}

University Campus STeP Ri

Slavka Krautzeka 83/A

51000 Rijeka, Croatia

Phone: +385 (51) 770447

Fax: +385 (51) 686166

www.intechopen.com

\section{InTech China}

Unit 405, Office Block, Hotel Equatorial Shanghai

No.65, Yan An Road (West), Shanghai, 200040, China 中国上海市延安西路65号上海国际贵都大饭店办公楼 405 单元

Phone: +86-21-62489820

Fax: $+86-21-62489821$ 
(C) 2011 The Author(s). Licensee IntechOpen. This chapter is distributed under the terms of the Creative Commons Attribution-NonCommercialShareAlike-3.0 License, which permits use, distribution and reproduction for non-commercial purposes, provided the original is properly cited and derivative works building on this content are distributed under the same license. 